\title{
A genetically encoded ratiometric calcium sensor enables quantitative measurement of the local calcium microdomain in the endoplasmic reticulum
}

\author{
Chen Luo ${ }^{1,2}$, Huiyu Wang ${ }^{1,2}$, Qi Liu ${ }^{1,2}$, Wenting He ${ }^{1}$, Lin Yuan ${ }^{1 \bowtie}$, Pingyong Xu ${ }^{1,2} \bowtie$ \\ ${ }^{1}$ Key Laboratory of RNA Biology, Institute of Biophysics, Chinese Academy of Sciences, Beijing 100101, China \\ ${ }^{2}$ College of Life Sciences, University of Chinese Academy of Sciences, Beijing 100049, China
}

Received: 6 September 2018 / Accepted: 15 November 2018 / Published online: 27 February 2019

\begin{abstract}
The local $\mathrm{Ca}^{2+}$ release from the heterogeneously distributed endoplasmic reticulum (ER) calcium store has a critical role in calcium homeostasis and cellular function. However, single fluorescent proteinbased ER calcium probes experience challenges in quantifying the ER calcium store in differing live cells, and intensity-based measurements make it difficult to detect local calcium microdomains in the ER. Here, we developed a genetically encoded ratiometric ER calcium indicator (GCEPIA1-SNAP ${ }_{E R}$ ) that can detect the real-time ER calcium store and local calcium microdomains in live cells. GCEPIA1-SNAP ${ }_{\mathrm{ER}}$ was located in the lumen of the ER and showed a linear, reversible and rapid response to changes in the ER calcium store. The GCEPIA1-SNAP ${ }_{\text {ER }}$ probe effectively monitored the depletion of the ER calcium store by TG or starvation treatment, and through its use we identified heterogeneously distributed calcium microdomains in the ER which were correlated with the distribution of STIM1 clusters upon ER calcium store depletion. Lastly, GCEPIA1-SNAP ${ }_{\mathrm{ER}}$ can be used to detect the ER calcium store by highthroughput flow cytometry and confers the ability to study the function of calcium microdomains of the ER.
\end{abstract}

Keywords Ratiometric calcium sensor, Local calcium microdomain, Endoplasmic reticulum

\section{INTRODUCTION}

The calcium stores in the endoplasmic/sarcoplasmic reticulum (ER) are heterogeneously distributed in most cells because of the uneven distribution of $\mathrm{ER} \mathrm{Ca}^{2+}$ binding proteins and immobile $\mathrm{Ca}^{2+}$-buffered solutions. Numerous protein processes may be affected by the

Chen Luo, Huiyu Wang and Qi Liu contributed equally to this work.

Electronic supplementary material The online version of this article (https://doi.org/10.1007/s41048-019-0082-6) contains supplementary material, which is available to authorized users.

$\triangle$ Correspondence: yuanlin@ibp.ac.cn (L. Yuan), pyxu@ibp.ac.cn (P. Xu) nonuniform distribution of $\left[\mathrm{Ca}^{2+}\right]_{\mathrm{ER}}$; for example, $\mathrm{Ca}^{2+}$-sensitive chaperones such as the lectin-like proteins calnexin and calreticulin (Carreras-Sureda et al. 2017; Clapham 2007). $\mathrm{Ca}^{2+}$ acts as a vital second messenger responding to a variety of environmental stimuli, and thus the heterogeneous release of $\mathrm{Ca}^{2+}$ from different parts of the ER undoubtedly could affect $\mathrm{Ca}^{2+}$-dependent processes including store-operated $\mathrm{Ca}^{2+}$ entry (SOCE), fusion of autophagosomes, cell motility or calcium transfer from the ER to mitochondria in the mitochondria-associated membrane (Ghislat and Knecht 2012; Rizzuto et al. 1998). Thus, it is important to monitor the dynamic change of calcium stores in these microdomains in living cells.

GCaMP proteins are widely used to image the change in intracellular calcium concentration of cells under different physiological stimuli. Subcellular-located 
GCaMPs were used to measure changes in the calcium concentration of different organelles. Currently, many probes based on GCaMPs have been developed for the measurement of ER calcium in live cells, and even in animals (Akerboom et al. 2012; Mao et al. 2008; Shigetomi et al. 2010; Tian et al. 2009). Usually, the measurements of ER calcium content are based on the fluorescence intensity of probes. However, the intensitybased measurements of the ER calcium store have several limitations. First, the fluorescence intensity is dependent on the expression levels of ER calcium probes, which differs among cells. Therefore, it is difficult to make cell-to-cell comparisons of the ER calcium store when studying a gene function and enormous amounts of data should be collected to minimize the variation. Second, the fluorescence intensity is also dependent on the local density of the ER calcium probe, which is determined by the local ER structures such as ER sheets. Thus, the intensity-based measurements face difficulty in precisely quantifying the calcium of microdomains. Third, time course quantification of the ER calcium store in live cells is challenging with intensitybased measurements, since the ER is highly dynamic, and the recorded calcium signal could be affected by defocus caused by the movement of the ER. Hence, it is confusing to judge whether the changed fluorescence signal reflects a change of true signal or simply defocus. Finally, imaging methods with intensity-based probes may be incapable of reliably quantifying and monitoring dynamic changes of local calcium in the ER domains of live cells.

All the above limitations could be overcome by normalizing probe expression levels with a ratiometric ERlocated calcium sensor. Zhao et al. developed ratiometric calcium probes with excitation and emission at distinct wavelengths in the $\mathrm{Ca}^{2+}$-free and $\mathrm{Ca}^{2+}$-bound states (Zhao et al. 2011). After that, Suzuki et al. optimized the blue-green emission ratiometric GECO and developed GEM-CEPIA1 $1_{\mathrm{ER}}$ with a low $\mathrm{Ca}^{2+}$ affinity and a large dynamic range for imaging ER calcium (Suzuki et al. 2014). However, there is a lethal weakness that the excited wavelength is around $400 \mathrm{~nm}$, which has severe phototoxicity to cells and is not suitable for live cell imaging (Icha et al. 2017; Wäldchen et al. 2015). Furthermore, the cytosolic GCaMP-based ratiometric probes, generally fused with red fluorescent proteins (FPs) such as mCherry (Cho et al. 2017), although avoiding the phototoxic effects of excitation light on cells, experience inherent disadvantages due to the aggregation property and slow maturation rate of mCherry. In addition, Förster resonance energy transfer (FRET) will decrease calcium signal and cannot be entirely avoided because of the spectral overlap between GCaMP and mCherry, despite a series of strategies adopted to reduce FRET (e.g., inserting rigid linkers into GCaMP and mCherry) (Cho et al. 2017).

In the current study, we developed a ratiometric ERlocated calcium sensor, GCEPIA1-SNAP ${ }_{E R}$, by fusing GCEPIA1 $1_{\mathrm{ER}}$, a mutant of GGaMP, with a SNAP-tag to dynamically quantify local calcium of ER microdomains in live cells. The SNAP-tag protein undergoes an irreversible self-labeling reaction to form a covalent bond with 06-benzylguanine (BG) derivatives, which can be modified with a variety of reporter molecules such as fluorophores, peptides, or oligonucleotides (Cole 2013). In this study, we used SNAP-Cell ${ }^{\circledR} 647-$ SiR to covalently couple SNAP-tag for two considerations. First, the farinfrared SNAP-647 uses $647 \mathrm{~nm}$ for illumination, which has less phototoxic than $405 \mathrm{~nm}$ illumination used for GEM-CEPIA1er when imaging live cells. Second, the emission spectrum of SNAP-647 is separated from GCEPIA $_{\text {ER }}$ and there is no FRET between GEM-CEPIA $1_{\text {ER }}$ and SNAP-647 that would affect calcium detection. Because the SNAP-tag is co-expressed in-frame with GCEPIA, and the fluorescence intensity of SNAP-647 does not change in different calcium concentrations, the SNAP-tag can be used for ratiometric measurement to indicate the location and expression level of GCEPIA. This tandem constructed probe facilitates pixel-wise ratiometric measurements of the calcium instead of fluorescence intensity alone. The local ER microdomains could be detected precisely by the ratiometric-based method.

\section{RESULTS}

\section{Characteristics of ER-located ratiometric calcium sensor GCEPIA1-SNAP ${ }_{\text {ER }}$}

The schematic construction of GCEPIA1-SNAP ${ }_{\mathrm{ER}}$ is shown in Fig. 1A. To examine the localization of the ratiometric probe, GCEPIA1-SNAP $\mathrm{ER}_{\text {was }}$ transfected into COS7 cells. The results showed that the KDEL retention signal peptide leads the fusion protein specifically to localization in the ER and that the SNAP tag labeled GCEPIA1 $1_{\mathrm{ER}}$ colocalized well with ER marker Sec61-mApple, indicating that the fusion of SNAP-tag does not affect the localization of the GCEPIA $1_{\mathrm{ER}}$ probe (Fig. 1B). We next examined the photochemical characteristics of GCEPIA1-SNAP ${ }_{\text {ER }}$. The excitation and emission spectra remain unchanged after fusing the SNAPtag with GCEPIA1 $1_{\mathrm{ER}}$. The spectra showed that the maximum excitation and emission wavelength of both probes are $402 / 502 \mathrm{~nm}$ and $512 \mathrm{~nm}$, respectively (Fig. 1C, D). Absorbance spectra of GCEPIA1-SNAP $\mathrm{ER}_{\mathrm{ER}}$ 
A GCEPIA1-SNAP ${ }_{\text {ER }}$

\begin{tabular}{|c|c|c|c|c|}
\hline RS20 & cpEGFP & CaM & SNAP & KDEL \\
\hline
\end{tabular}

B
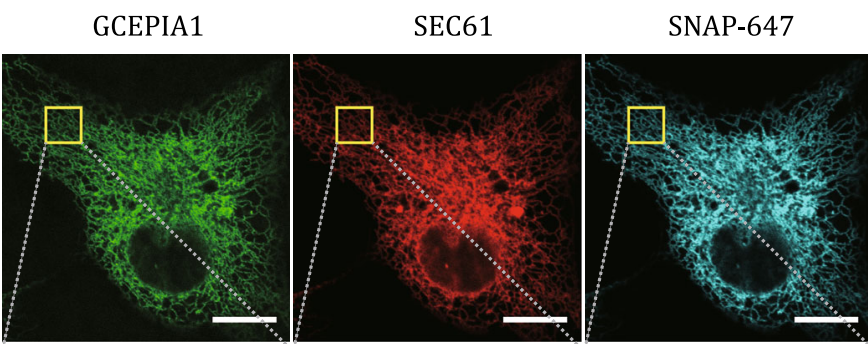

GCEPIA1+SEC61

SNAP-647+SEC61
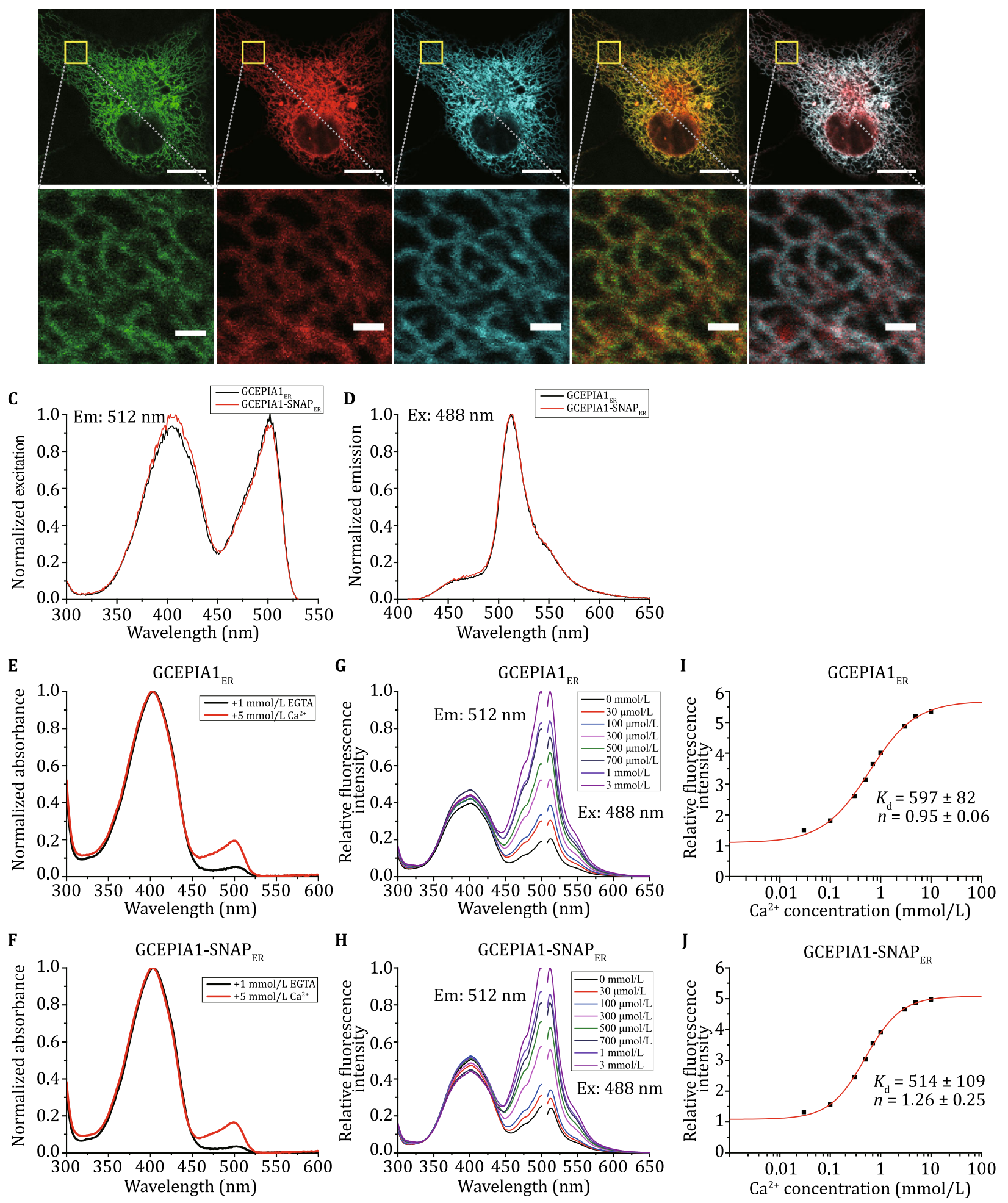
4Fig. 1 Construction and characteristics of GCEPIA1-SNAP ER $_{\text {. }}$

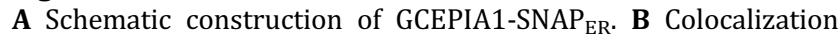
of GCEPIA1-SNAP ${ }_{\mathrm{ER}}$ with ER marker Sec61-mApple in COS7 cells. Green: GCEPIA1; Red: Sec61-mApple; Cyan: SNAP-647; Yellow: Merge of GCEPIA1 with Sec61-mApple; White: Merge of SNAP-647 with Sec61-mApple. Images within the rectangle that showed in the upper panel were zoomed in and shown in the lower panel. Scale bars: $10 \mu \mathrm{m}$ (upper panel) and $1 \mu \mathrm{m}$ (lower panel). CJ Properties of GCEPIA1-SNAP $\mathrm{ER}_{\mathrm{ER}}$ in vitro. Normalized excitation (C) and emission (D) spectra of GCEPIA1-SNAP ${ }_{\mathrm{ER}}$ (red) and GCEPIA1 $1_{\mathrm{ER}}$ (black). Normalized absorbance spectra of GCEPIA1 $1_{\mathrm{ER}}$ (E) and GCEPIA1-SNAP ${ }_{\mathrm{ER}}(\mathbf{F})$ in $\mathrm{Ca}^{2+}$-containing $\left(5 \mathrm{mmol} / \mathrm{L} \mathrm{CaCl}_{2}\right.$, red) or $\mathrm{Ca}^{2+}$-free (1 mmol/L EGTA, black) buffer. The spectra were normalized by the maximum intensity. Spectral titration curve of GCEPIA1 $_{\mathrm{ER}}$ (G) and GCEPIA1-SNAP ${ }_{\mathrm{ER}}(\mathbf{H})$ with various $\mathrm{Ca}^{2+}$ concentrations. The fluorescence intensity was normalized by the maximum intensity. For excitation spectra, fluorescence intensity at $512 \mathrm{~nm}$ was obtained for GCEPIA1 $1_{\mathrm{ER}}$ and GCEPIA1-SNAP ${ }_{\mathrm{ER}}$. For emission spectra, GCEPIA $1_{\mathrm{ER}}$ and GCEPIA1-SNAP ${ }_{\mathrm{ER}}$ were excited at $488 \mathrm{~nm}$. Fitted Hill plot curves for $\mathrm{Ca}^{2+}$ titration of GCEPIA1 $1_{\mathrm{ER}}$

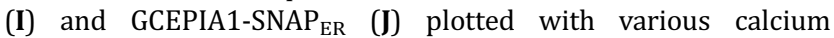
concentrations. The fluorescence intensity was normalized to the minimum intensity

and the original GCEPIA $1_{\mathrm{ER}}$ in $\mathrm{Ca}^{2+}$-containing or $\mathrm{Ca}^{2+}$-free buffer also showed the similar tendency (Fig. 1E, F). There is no significant difference of the spectral titration curves at gradient calcium concentrations between GCEPIA1 $1_{\mathrm{ER}}$ and GCEPIA1-SNAP ${ }_{\mathrm{ER}}$ (Fig. 1G-J), as are the calculated calcium-binding constants, dynamic ranges, $K_{\mathrm{d}}$ values and the Hill coefficients (Supplementary Table S1). These characteristics

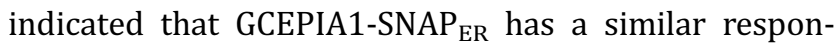
siveness and dynamic range to calcium compared with GCEPIA1 $1_{\text {ER }}$.

\section{Application of GCEPIA1-SNAP ${ }_{\mathrm{ER}}$ to detect the whole-cell ER calcium store}

We then detected the ER calcium store in different cells using GCEPIA1-SNAP $\mathrm{ER}$-transfected COS7 cells. The expression level of GCEPIA1-SNAP $\mathrm{ER}_{\mathrm{E}}$ varies greatly between different cells due to varying transfection efficiency (three selected cells shown in Fig. 2A). The basal ER calcium store was measured by detecting the green calcium signal of GCEPIA1 and normalizing the expression level of the red SNAP signal (Fig. 2A, 3rd row, $\mathrm{T}_{0}$ time point). Different cells showed variant basal ER calcium stores (Fig. 2D, $\mathrm{T}_{0}$ time point).

To prove that GCEPIA1-SNAP $\mathrm{ER}_{\text {R }}$ can monitor the dynamic ER calcium store, we treated cells with thapsigargin (TG), the most widely used noncompetitive inhibitor of the sarco/endoplasmic reticulum $\mathrm{Ca}^{2+}$. ATPase (SERCA), to deplete the ER calcium store; we then measured ER calcium at different time points. Laser scanning confocal microscope images at different time points after TG stimulation showed that the green GCEPIA1 fluorescence was decreased gradually while the red SNAP-dye fluorescence was decreased slightly due to photo-bleaching (Fig. 2A-C).

\section{The heterogeneous distribution of local ER calcium store}

Previously, GCEPIA $1_{\mathrm{ER}}$ was used to detect the entire ER calcium store by depleting the ER calcium with TG and calculating the maximum fluorescence decrease, which was normalized by the expression level $\left(\Delta F / F_{0}\right)$. However, the intensity-based method of $\Delta F / F_{0}$ is incapable of monitoring ER-dynamic change in microdomains of live cells, because local $F_{0}$ are different for each local ER structure and because it is impossible to deplete local ER calcium store, since ER is a highly dynamic continuous network structure. As shown in Fig. 3A, the local ER calcium distribution was heterogeneous in basal COS7 cells. Upon TG stimulation, the total fluorescence of

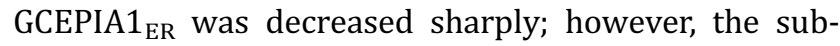
cellular distribution of GCEPIA1 $1_{\mathrm{ER}}$ was nonuniform in the ER and showed many aggregated clusters surrounding the nucleus or underneath the plasma membrane (Fig. 3A). Moreover, the calcium signals with TG treatment were higher at some pixels than those without TG treatment (Fig. 3B). These results suggested that ER structures are dynamic and reorganized to form microdomains. Apparently, it is impossible to detect the local calcium microdomains using the GCEPIA $1_{\mathrm{ER}}$ probe due to the ER movement and local aggregation of the probes.

\section{Detection of local ER calcium microdomains by GCEPIA1-SNAP ${ }_{\text {ER }}$}

GCEPIA1-SNAP $_{\text {ER }}$ was then applied to study calcium microdomains in COS7 cells. Since SNAP and GCEPIA1 are expressed as a fusion protein, local calcium at any ER microdomain can be precisely represented by normalizing local expression of the probe to the far-red signal of SNAP. The distribution of intracellular calcium in cells was not uniform (Fig. 4A), and this heterogeneity was related to ER structure: higher calcium regions were mainly distributed in ER near the nucleus (mainly in ER sheet structures), and the calcium concentration gradually descended to the periphery (Fig. 4B). The results may suggest that the ER sheet structure needs higher calcium content to maintain special physiological functions, such as the synthesis, folding and transport process of membrane and secretory proteins. The profile of a yellow line has been drawn in Fig. 4A, which represents the value of GCEPIA1 intensity (black line), and the ratio of GCEPIA1/SNAP ${ }_{\text {ER }}$ (red line) at each pixel point, 
A

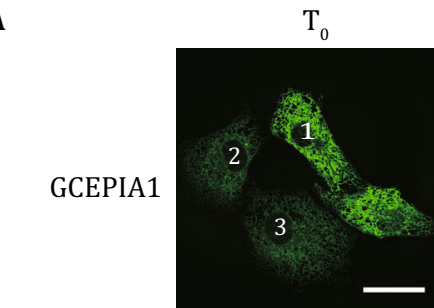

SNAP-647
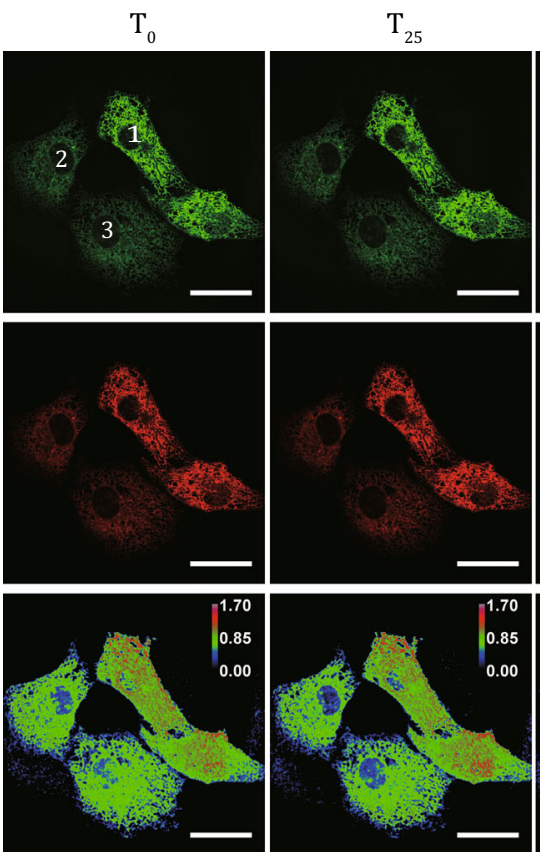

B

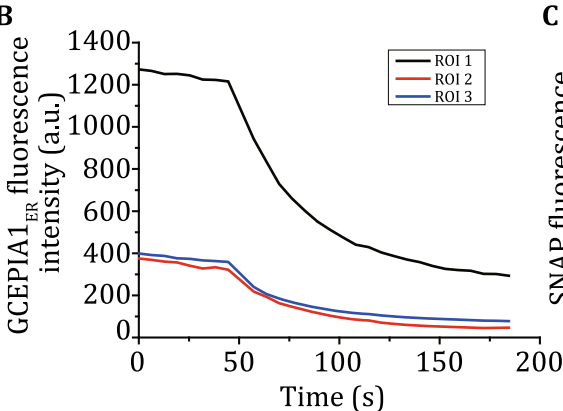

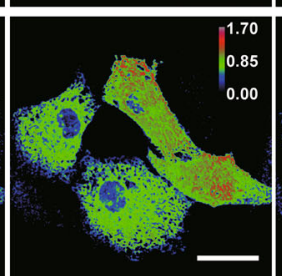
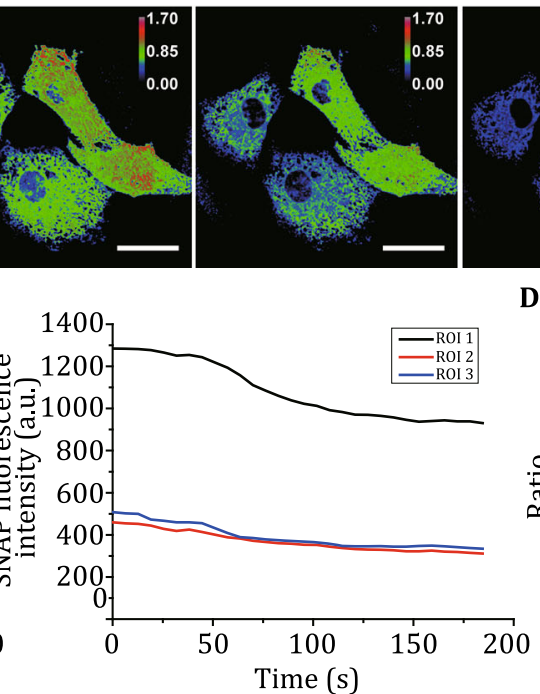
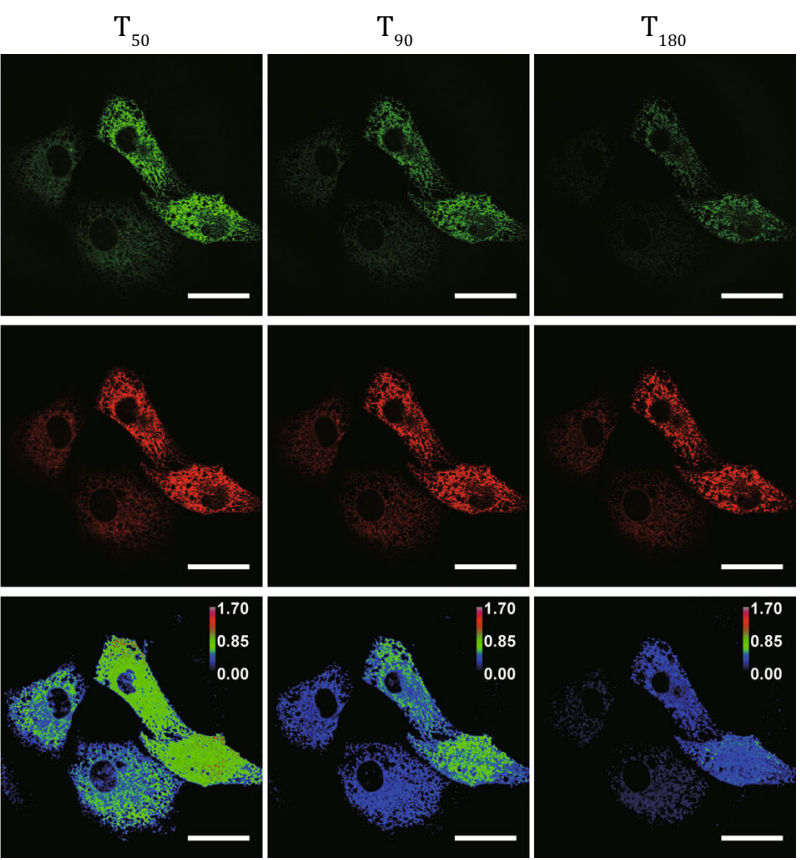

D

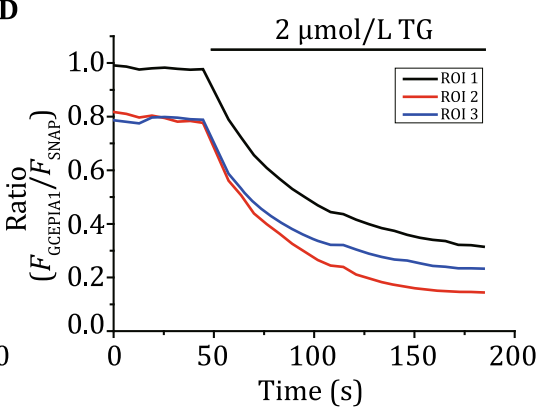

Fig. 2 Detection of the ER calcium store by GCEPIA1-SNAP ${ }_{\text {ER }}$ A COS7 cells were transfected with GCEPIA1-SNAP ER The images were taken by laser scanning confocal microscopy every $5 \mathrm{~s}$, and TG was performed at the time point of $50 \mathrm{~s}$. The images at $0,25,50,90$ and $180 \mathrm{~s}$ are shown. Intensity of GCEPIA1 is shown in the top panel (green), and the signal of SNAP-647 is shown in the middle panel (red). Pseudocolor ratiometric images represent the relative calcium level (bottom panel). Scale bars, $30 \mu \mathrm{m}$. The fluorescence intensities of GCEPIA1 and SNAP-647 in response to TG are shown in B and C. The selected three ROIs are shown in A. D Ratio of GCEPIA1 over SNAP to indicate the calcium dynamic change in response to $2 \mu \mathrm{mol} / \mathrm{L} \mathrm{TG}$. The initial value represents the ER calcium store

with or without TG treatment, are shown in Fig. 4B and 4C, respectively. TG stimulation-induced ER store depletion changed the local ER calcium distribution (Fig. 4). Apparently, compared to the GCEPIA1 intensitybased detection, GCEPIA1/SNAP using local normalization of expression level showed different local ER calcium profiles for both basal and ER calcium depletion conditions (Fig. 4B, 4C), and exhibited an improved ability to discriminate the differences of ER calcium signals between neighboring microdomains, especially in the regions with low calcium signals (Fig. 4B).

\section{Relationship between local calcium microdomains and distribution of STIM1 cluster}

Simultaneously, imaging the ER local calcium and other physiological processes could supply valuable information on how local calcium domains regulate important biological processes. Here, we aimed to simultaneously measure the local ER calcium microdomain and the clustering of STIM1, an important process in which the ER calcium sensor STIM1 aggregates and translocates at the ER-plasma membrane junction to oligomerize and activate the calcium release-activated calcium (CRAC) 
A

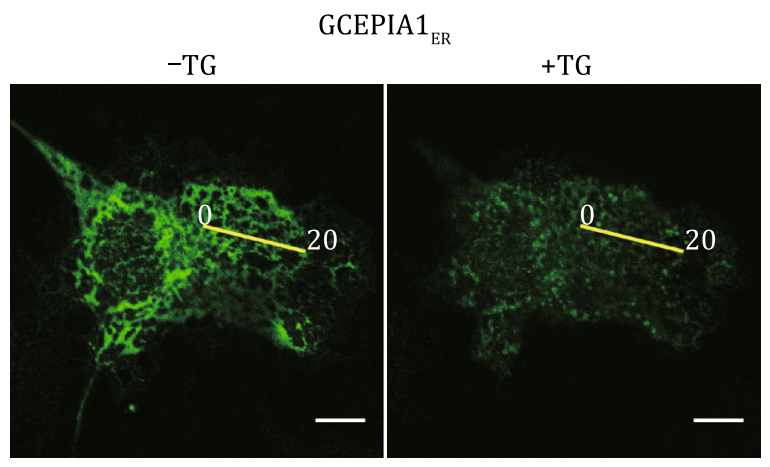

C

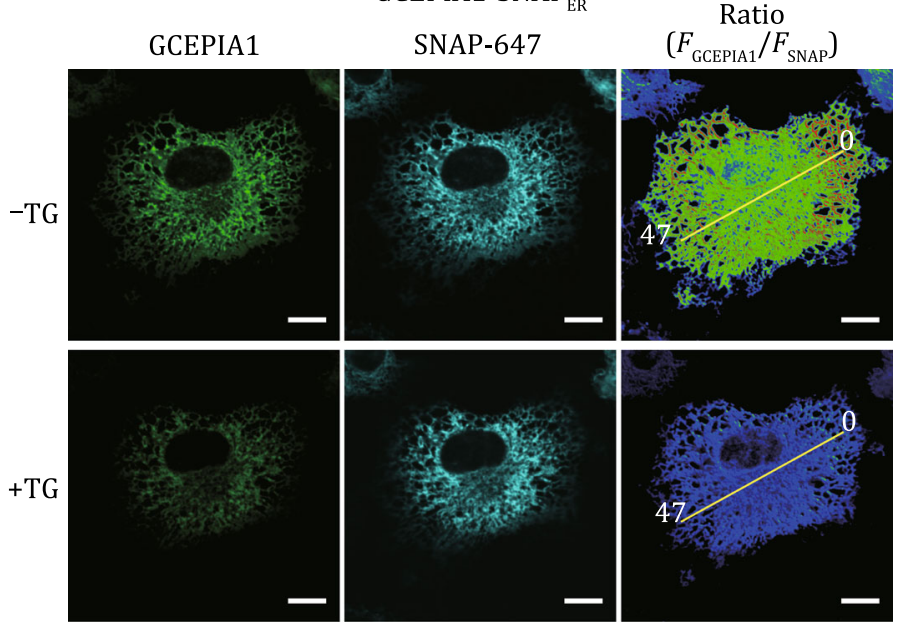

B

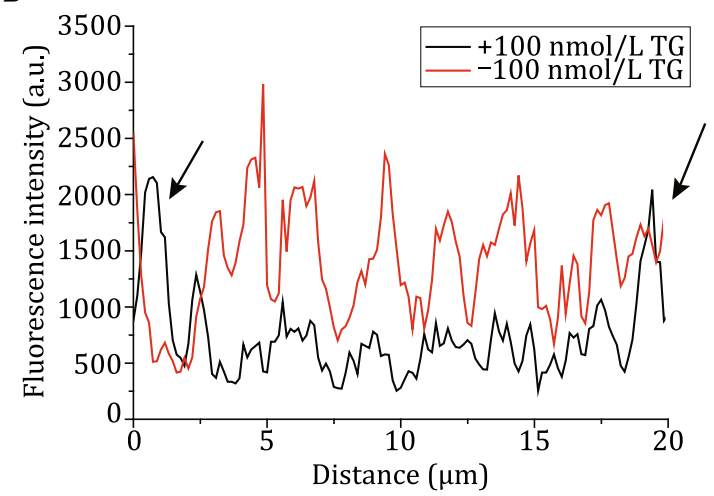

D

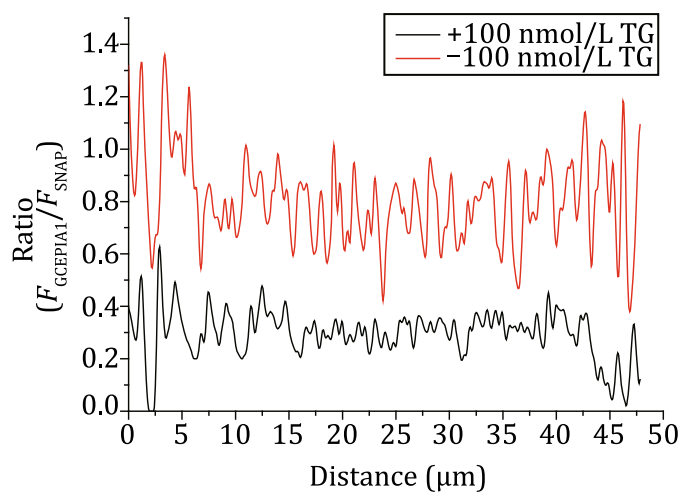

Fig. 3 The limitation of GCEPIA1 $1_{\mathrm{ER}}$ in ER calcium microdomain detection. A The calcium labeled by GCEPIA1 $1_{\mathrm{ER}}$ probe was imaged by laser scanning confocal with or without TG stimulation. Scale bars, $10 \mu \mathrm{m}$. B Fluorescence intensity of each pixel on the line which has been drawn in A. Arrows mark the pixels of fluorescence intensity with TG treatment higher than that without TG stimulation. C The calcium labeled by GCEPIA1-SNAP $\mathrm{ER}$ probe was imaged by laser scanning confocal with or without TG stimulation. Scale bars, $10 \mu \mathrm{m}$. D Fluorescence intensity of each pixel on the line which has been drawn in $\mathbf{C}$

channel upon $\mathrm{ER} \mathrm{Ca}^{2+}$ depletion (Liou et al. 2005; Park et al. 2009; Peinelt et al. 2006; Prakriya et al. 2006; Zhang et al. 2005). A previous study showed that the number of STIM1 clusters is negatively correlated with the total ER calcium store (Suzuki et al. 2014). However, the relationship between the precise distribution of STIM1 clusters and the ER calcium microdomains after $\mathrm{ER} \mathrm{Ca}^{2+}$ depletion has not been resolved. We measured local ER calcium using GCEPIA1-SNAP $\mathrm{ER}_{\mathrm{ER}}$ and simultaneously imaged STIM1 dynamics using STIM1mOrange2. After the depletion of ER calcium, the number of STIM1 puncta increased obviously, which showed in ours (Fig. 5A, with or without TG treatment) and the previous results (Suzuki et al. 2014). We also found that although the total ER calcium is greatly decreased after TG treatment, the distribution of ER calcium is still not uniform, which indicates the existance of ER calcium microdomains (Fig. 5A). Furthermore, most of the positions of STIM1 puncta are highly correlated with the region with a relatively high calcium concentration, which phenomenon is not reported previously (Fig. 5B). The biological significance of these ER calcium microdomains need to be studied in the future.

\section{The content of the ER calcium store decreased under starvation}

The ER calcium store is precisely regulated by different types of calcium channels and pumps: inositol trisphosphate receptor $\left(\mathrm{IP}_{3} \mathrm{R}\right)$ binds to $\mathrm{IP}_{3}$ and allows calcium to exit the ER, while the sarco-endoplasmic reticulum $\mathrm{Ca}^{2+}$-ATPase (SERCA) pump transports $\mathrm{Ca}^{2+}$ from the cytosol to refill the ER in an ATP-consuming fashion (Stutzmann 2005). For the reason that the SERCA pump calcium transport requires much energy, we proposed that the activity of the SERCA will be decreased under starvation and lead to the reduction of the ER calcium store.

To prove this hypothesis, MEF cells were transfected

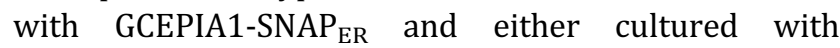


A

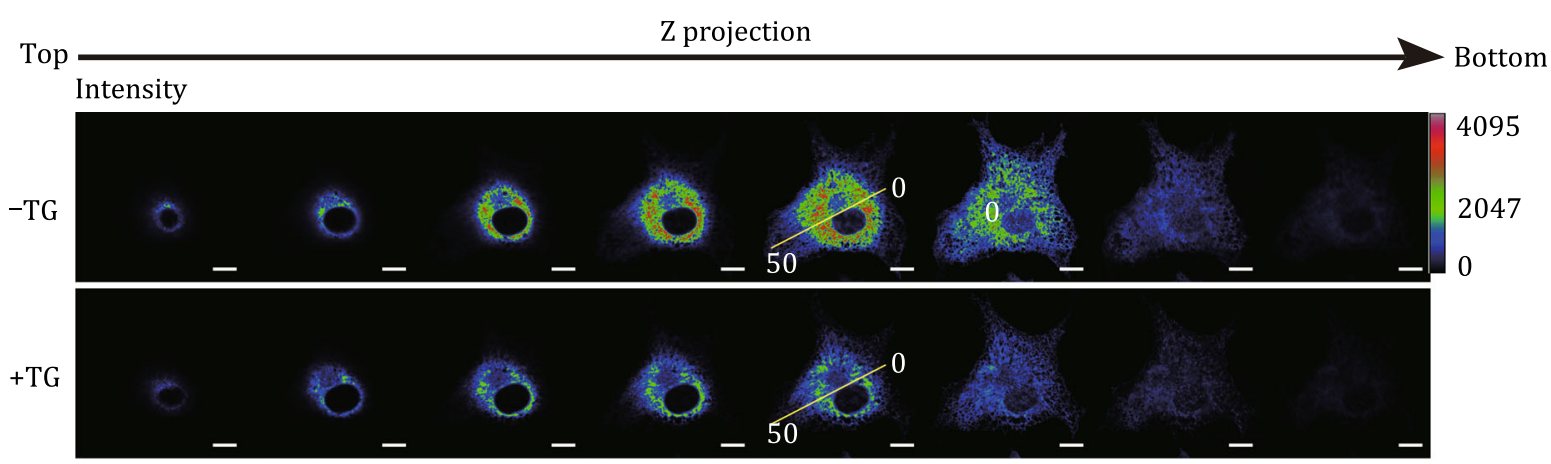

Ratio (GCEPIA1/SNAP)

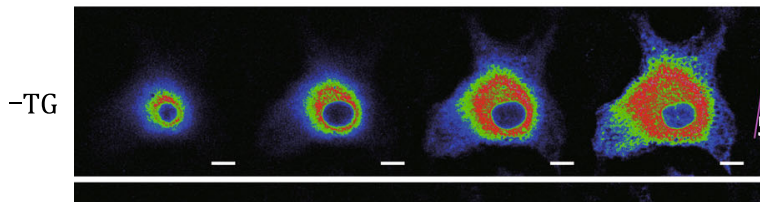

$+\mathrm{TG}$

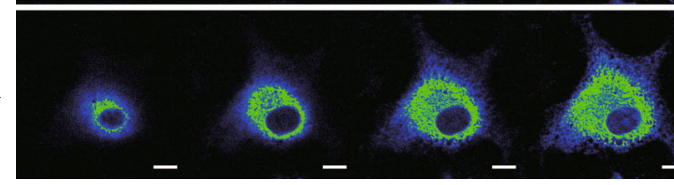

B

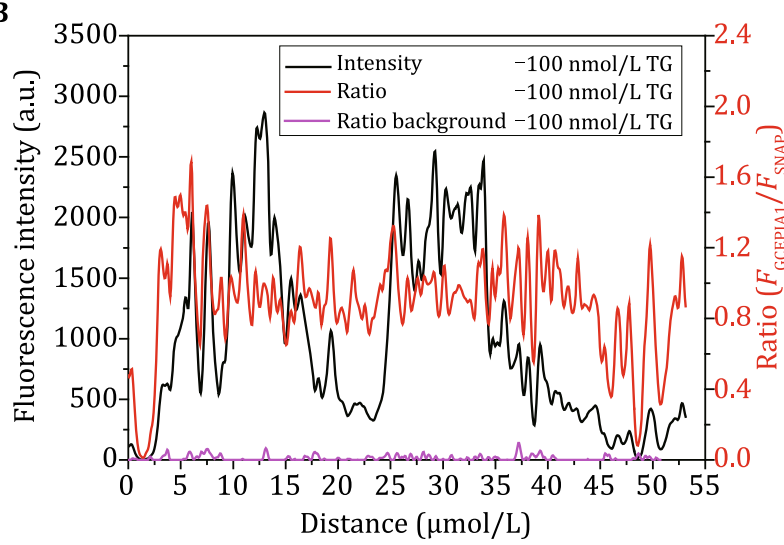

C

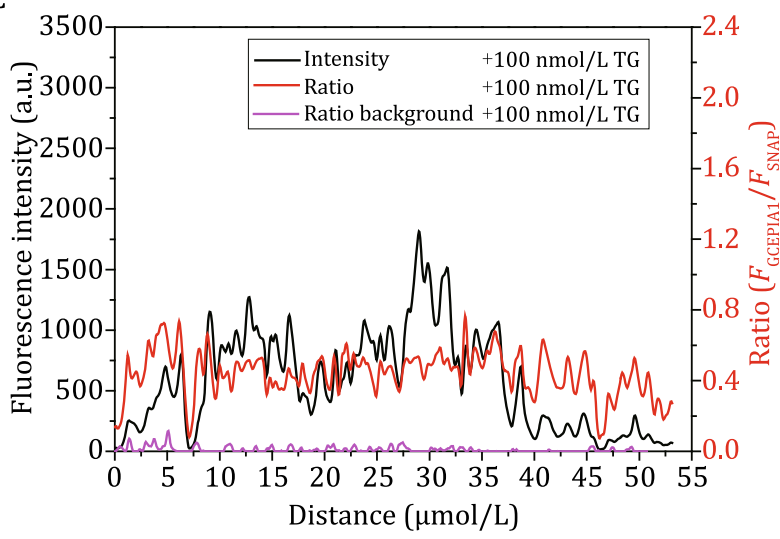

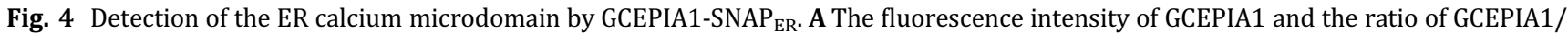
SNAP through the $z$-axis in COS7 cells, which were stimulated with or without TG treatment, are shown. B, C Profiles of the intensity of GCEPIA1 and the ratio of GCEPIA1/SNAP and background at each pixel of the line drawn in (A) without (B) or with TG treatment (C)

complete culture medium or starved with Hanks buffer for $2 \mathrm{~h}$. Indeed, the calcium content of the ER was significantly reduced under starvation compared with that of the nonstarvation group (Fig. 6A). To further confirm this result, Fura-2/AM was employed to detect the calcium released from the ER with TG treatment in calcium-free buffer. The results showed that starvation impaired the ER calcium store (Fig. 6B, C), which is consistent with the results detected by GCEPIA1$\mathrm{SNAP}_{\mathrm{ER}}$. To detect whether the reduction of the ER calcium store is caused by the decrease of SERCA activity, the microsome was purified from MEF cells and the activity of SERCA was analyzed in vitro. Results showed that the activity of SERCA was significantly reduced upon starvation treatment (Fig. 6D), which revealed that sufficient intracellular energy is an essential factor for maintaining the ER calcium storage.

\section{GCAMPIA1-SNAP ${ }_{E R}$ for high-throughput detection of ER calcium content by flow cytometry}

MEF cells were transiently transfected with GCEPIA1SNAP $_{\text {ER }}$ sensor and treated with or without $2 \mu \mathrm{mol} / \mathrm{L}$ TG. The transfected positive cells were gated and analyzed by flow cytometry (Fig. 7A, B). Figure 7C shows that the population of the TG-treated group was leftshifted, which indicated that the calcium content was decreased as expected. Thus, high throughput screening of the effects of stimuli on ER calcium content can be performed using GCEPIA1-SNAP ${ }_{\mathrm{ER}}$. 
A
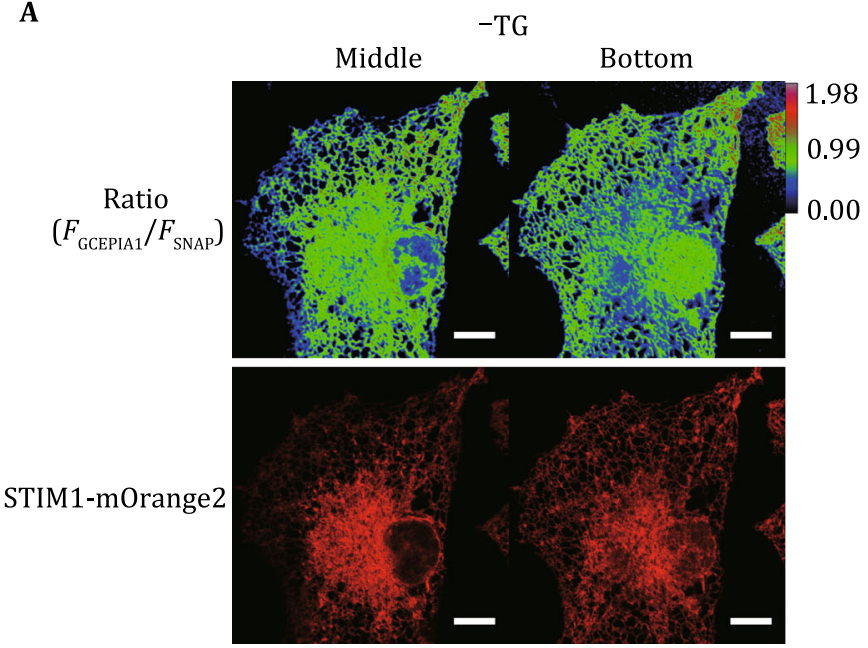

B

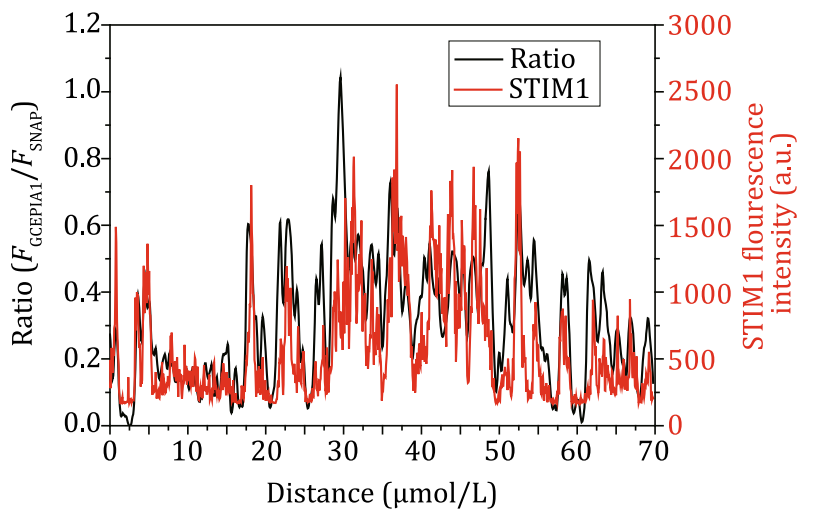

Fig. 5 Relationship between content of calcium microdomain and distribution of STIM1 cluster. A Z projection images of STIM1mOrange2 and the pseudocolor ratiometric images of GCEPIA1 ${ }_{\mathrm{ER}} / \mathrm{SNAP}$ of COS7 cells without or with TG treatment. Scale bars, $10 \mu \mathrm{m}$. B Relationship between the distributions of STIM1 clusters and the calcium content of each pixel on the line, which has been drawn in A

\section{DISCUSSION}

In the current study, we developed a new ratiometric ER calcium probe GCEPIA1-SNAP $\mathrm{ER}_{\mathrm{ER}}$. Compared to currently used fluorescence intensity-based ER calcium probes, GCEPIA1-SNAP ER provides several advantages.

First, GCEPIA1-SNAP ${ }_{\mathrm{ER}}$ has the ability to measure the ER calcium store more accurately, and it can measure the basal ER calcium store. Previously, the fluorescence intensity was normalized by dividing the average of the total fluorescence values $\left(F_{0}\right)$ for each cell at resting state, and the change of ER calcium store was calculated as $\Delta F / F_{0}=\left(F_{n}-F_{0}\right) / F_{0}$, where $F_{n}$ is the total fluorescence value for each cell at different time points (Fig. 2D) (Koldenkova and Nagai 2013; Suzuki et al. 2014). However, besides the expression level of the probe, the resting fluorescence $F_{0}$ is also affected by the dynamic exchange between cellular and extracellular calcium concentrations; thus, normalized fluorescence intensity would also eliminate the information of the initial calcium concentration at resting state. Moreover, the major limitation of the intensity-based method is the dependence on drug stimulation to empty the ER calcium - the method cannot be used to directly monitor the basal ER calcium store at the resting state without TG stimulation. Differently from the currently used fluorescence protein intensity-based ER calcium sensor, our ratiometric probe GCEPIA1-SNAP ${ }_{\mathrm{ER}}$ utilizes a SNAPtag for direct calibration of probe expression and can be used for measuring the basal ER calcium store. Additionally, ratiometric GCEPIA1-SNAP ${ }_{\mathrm{ER}}$ can calibrate the deviations caused by defocusing of the focal plane, transfection/translation efficiency differences, dynamic movement of the ER, and others. Furthermore, compared to two-fluorescence protein dual reporter systems, GCEPIA1-SNAP ${ }_{\mathrm{ER}}$ minimizes the problem of maturation because the chromophore of the Alexa 647 dye has already been formed before binding to the SNAP-tag.

Second, GCEPIA1-SNAP ${ }_{\text {ER }}$ enables simultaneous detection of the ER calcium store and other cellular dynamics in one live cell. Due to the influence of the 
A

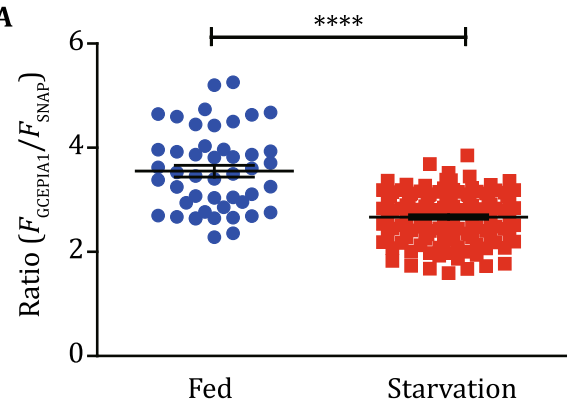

C

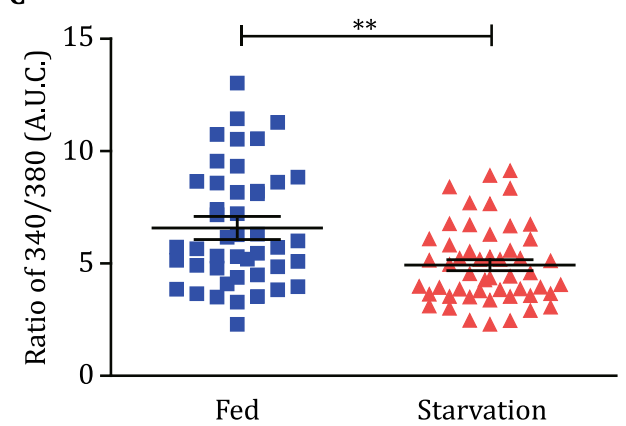

B
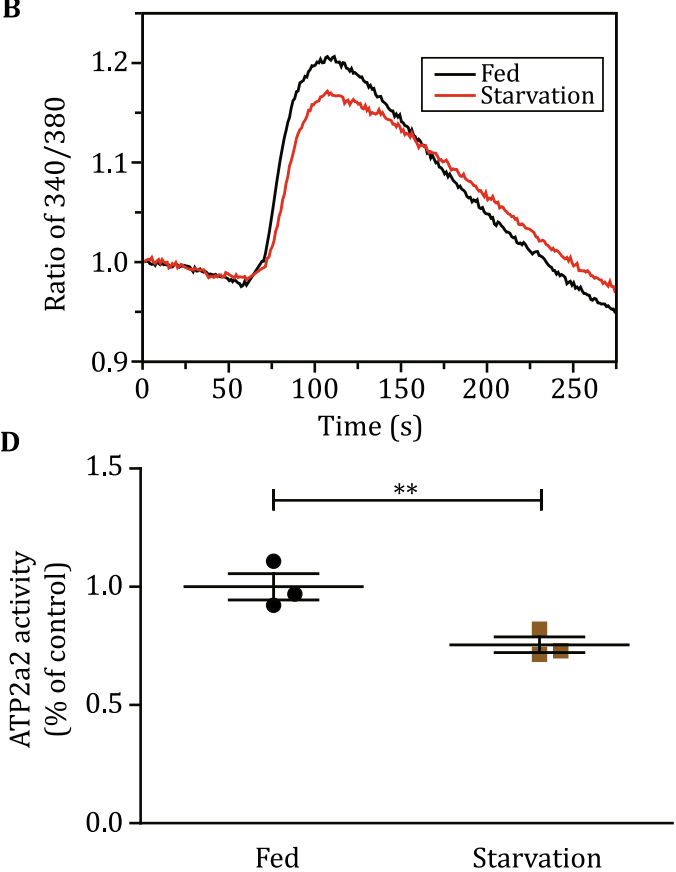

Fig. 6 Starvation treatment decreases the ER calcium store. A MEF cells were transfected with GCEPIA1-SNAP ER $_{\text {and }}$ either cultured with complete culture medium or starved for $2 \mathrm{~h}$ with Hanks buffer. The ratio of GCEPIA1 to SNAP, which indicated the ER calcium store, is significantly decreased upon starvation treatment. Number of cells analyzed: fed, $n=47$; starvation, $n=125$. B The average curve of Fura-2/AM under fed or starvation conditions stimulated with $2 \mu \mathrm{mol} / \mathrm{L}$ TG (number of cells analyzed: fed, $n=46$; starvation, $n=51$ cells). C Area under the curve (A.U.C.) of ER calcium store depletion with TG treatment. D ATPase activity of microsomes from MEF cells cultured in complete culture medium or starved in Hanks buffer. Experiments were repeated three times. Data are represented as the mean \pm S.D. ${ }^{* *} P<0.05,{ }^{* * * *} P<0.0001$

variant expression level of probe, early methods for measuring and comparing ER calcium stores of different cells cannot directly read the calcium store signal, which must be calculated by measuring the reduction of calcium fluorescence signal after completely depleting the ER with TG. Since TG affects the function of cells, it is not possible to simultaneously detect the ER calcium store and other functions in one live cell. In the current study, since GCEPIA1-SNAP $\mathrm{ER}_{\text {in }}$ capable of calibrating the difference in expression amount in real time and reporting the entire ER calcium content, GCEPIA1SNAP $_{\mathrm{ER}}$ enables simultaneous detection of the ER calcium store and other cellular dynamics in one live cell. Furthermore, GCEPIA1-SNAP ${ }_{\text {ER }}$ can be easily labeled with specific BG dyes with separate emission spectra, which enables multicolor imaging of the ER calcium store and other biological processes.

Third, GCEPIA1-SNAP ${ }_{\mathrm{ER}}$ enables dynamic detection of the local ER calcium microdomains in live cells. Using fluorescence intensity-based ER calcium probes, the local ER calcium cannot be normalized by local $F_{0}$, as ER is highly dynamic and, accordingly, local probe density changes quickly. Thus, it is impossible that fluorescence intensity-based ER calcium probes can be used for detecting local calcium microdomains. In our study, the tandem fusion of GCEPIA1 $1_{\mathrm{ER}}$ with a SNAP-tag assures two proteins localized in the same pixel, which makes it feasible to perform local calibration and monitor the real-time ER calcium microdomains. Moreover, the formation process of dynamic clusters of STIM1 has been successfully imaged simultaneously with local ER calcium store in live cells in the current study.

\section{EXPERIMENTAL PROCEDURES}

\section{Plasmid construction}

The pAAV-H1-GCEPIA1 $1_{\mathrm{ER}}$ plasmid is a gift from Dr. Liangyi Chen at Peking University. SNAP-tag was fused in frame with GCEPIA1 containing the ER retention signal sequence (KDEL) using primers 1-4 (Supplementary Table S2).

For prokaryote expression of GCEPIA1-SNAP ${ }_{\text {ER }}$ and GCEPIA1 $_{\mathrm{ER}}$, we cloned GCEPIA1-SNAP $\mathrm{ER}_{\mathrm{ER}}$ or GCEPIA1 $1_{\mathrm{ER}}$ into the pRset (A) vector using primers $55 \mathrm{~s}, 55 \mathrm{a}, 56 \mathrm{~s}$, and $61 \mathrm{~s}$ with the template of pAAV-H1-GCEPIA1SNAP $_{\mathrm{ER}}$ or PAAV-H1-GCEPIA1 $1_{\mathrm{ER}}$. 
A
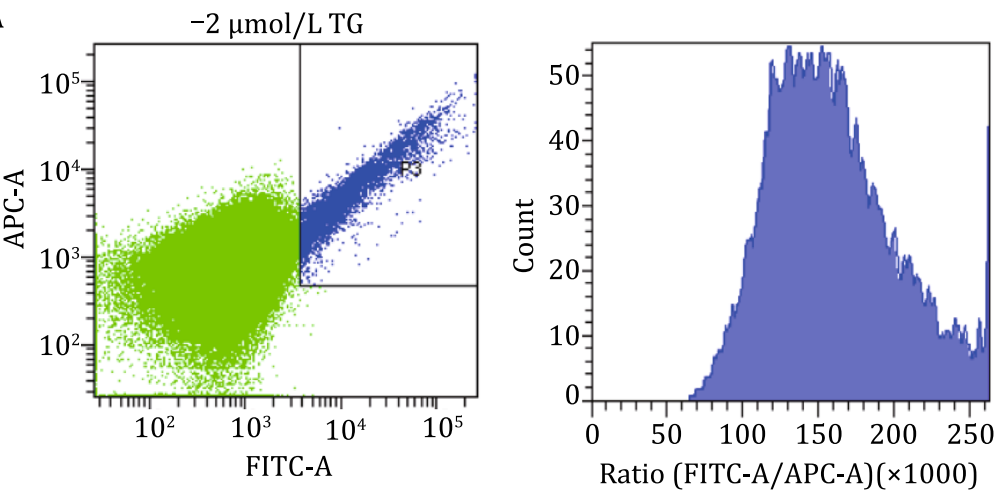

B
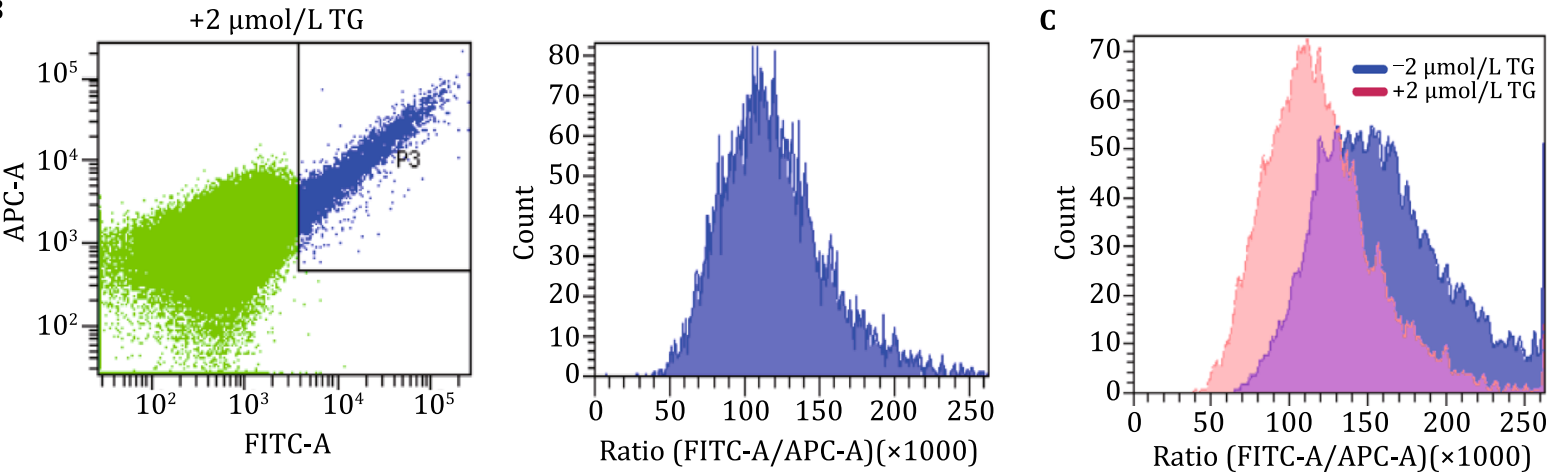

Fig. 7 High throughput identification of the ER calcium store by flow cytometry. A Intensity of ER calcium store of MEF cells transfected with GCEPIA1-SNAP ${ }_{\mathrm{ER}}$ and without TG treatment. B Intensity of ER calcium store of MEF cells transfected with GCEPIA1-SNAP ${ }_{\mathrm{ER}}$ and with TG treatment. C Distribution of calcium content in MEF cells with TG treatment is left-shifted. APC-A, area of allophycocyanin; FITC-A, area of fluorescein isothiocyanate

\section{Prokaryotic expression}

BL21 (DE3) competent E. coli cells (TransGen Biotech, China) were transfected with plasmids and incubated on LB Agar ampicillin $(100 \mu \mathrm{g} / \mathrm{mL})$ plates for $12 \mathrm{~h}$ at $37{ }^{\circ} \mathrm{C}$. Single colonies were separately inoculated in $10 \mathrm{~mL}$ of LB broth medium and were left overnight at $37{ }^{\circ} \mathrm{C}$ with moderate shaking and then induced with IPTG $(0.8 \mathrm{mg} / \mathrm{mL})$ for $16-24 \mathrm{~h}$ at $16{ }^{\circ} \mathrm{C}$ for target protein expression. The cells were resuspended in binding buffer $(50 \mathrm{mmol} / \mathrm{L}$ Tris, $500 \mathrm{mmol} / \mathrm{L} \mathrm{NaCl}, 10 \mathrm{mmol} / \mathrm{L}$ imidazole). After cell lysis with sonication, recombinant proteins were purified using a Ni-NTA His-Bind resin (Qiagen) followed by a gel filtration step with the Superdex 200 increase 10/300 GL column (GE Healthcare, Sweden). For further analysis, the purified proteins were concentrated via ultrafiltration and then resuspended in $\mathrm{KCl} / \mathrm{MOPS}$ buffer $(130 \mathrm{mmol} / \mathrm{L} \mathrm{KCl}$, $50 \mathrm{mmol} / \mathrm{L}$ MOPS, $\mathrm{pH}$ 7.2).

\section{In vitro spectroscopic determination}

$\mathrm{Ca}^{2+}$ titration curves of GCEPIA1 $1_{\mathrm{ER}}$ and GCEPIA1$\mathrm{SNAP}_{\mathrm{ER}}$ were obtained by adding small aliquots of $\mathrm{CaCl}_{2}$ to probes in a $\mathrm{KCl} / \mathrm{MOPS}$ buffer containing 0 or
$1 \mathrm{mmol} / \mathrm{L}$ EGTA at $\mathrm{pH}$ 7.2. The free calcium concentration was calculated using MaxChelator Version 1.2. Fluorescence intensity was measured with a spectrophotometer (F7000, HITACHI, Japan) for protein at $488 \mathrm{~nm}$ excitation/512 $\mathrm{nm}$ emission wavelengths. The obtained $\mathrm{Ca}^{2+}$-fluorescence intensity relationship was fitted by the Hill plot equation: $F=F_{\text {min }}+\left(F_{\max }-F_{\min }\right)$ $\times\left(\left[\mathrm{Ca}^{2+}\right]_{\text {free }}\right)^{n} /\left[\left(\left[\mathrm{Ca}^{2+}\right]_{\text {free }}\right)^{n}+\left(K_{\mathrm{d}}\right)^{n}\right] . K_{\mathrm{d}}$ denotes dissociation constant, which also means the calcium concentration with half of the probe binding to $\mathrm{Ca}^{2+}$. The $n$ represents the Hill coefficient. $F_{\min }$ refers to the value of emission peak of the lowest calcium concentration, and $F_{\text {max }}$ refers to the value of emission peak of the highest calcium concentration. The dynamic range of the indicator was calculated as the ratio of maximum and minimum fluorescence intensity $\left(F_{\max } / F_{\min }\right)$. The absorbance spectra were measured by a spectrophotometer (VARIOSKAN FLASH, Thermo Fisher Scientific, USA) for each protein in $\mathrm{KCl} / \mathrm{MOPS}$ buffer with $1 \mathrm{mmol} / \mathrm{L}$ EGTA or with $5 \mathrm{mmol} / \mathrm{L}$ $\mathrm{CaCl}_{2}$.

\section{Cell culture}

COS7 cells and MEF cells were cultured in DMEM (Invitrogen) supplemented with $10 \%$ fetal bovine serum 
(HyClone, Logan, UT) in an incubator at $37{ }^{\circ} \mathrm{C}$ with $5 \%$ $\mathrm{CO}_{2}$. For $\mathrm{Ca}^{2+}$ imaging, the cells were plated on $35 \mathrm{~mm}$ glass-bottom dishes (D35-10-1-N, Cellvis).

\section{Cellular labeling}

SNAP-Cell ${ }^{\circledR}$ 647-SiR was purchased from New England Biolabs (NEB) and cell labeling was performed according to the manufacturer's instructions. Briefly, the cells were transfected with GCEPIA1-SNAP ${ }_{\text {ER }}$ plasmid. After $24 \mathrm{~h}$, the cells were incubated with diluted SNAP-Cell ${ }^{\circledR}$ $647-\mathrm{SiR}(25 \mathrm{nmol} / \mathrm{L})$ labeling solution and incubate at $37{ }^{\circ} \mathrm{C}, 5 \% \mathrm{CO}_{2}$ for $30 \mathrm{~min}$.

\section{Microsome isolation}

MEF cells were collected and homogenized using a Dounce Tissue Grinder (Thermo Fisher) in ice-cold homogenized buffer $(20 \mathrm{mmol} / \mathrm{L}$ HEPES, $250 \mathrm{mmol} / \mathrm{L}$ sucrose, $1 \mathrm{mmol} / \mathrm{L}$ EDTA, pH 7.4) containing protease inhibitor cocktail (Sigma). The homogenates were centrifuged at $1000 \mathrm{~g}$ for $10 \mathrm{~min}$ at $4{ }^{\circ} \mathrm{C}$. Then, the supernatant was collected and centrifuged at $8000 \mathrm{~g}$ for $20 \mathrm{~min}$ at $4{ }^{\circ} \mathrm{C}$. The supernatant was ultracentrifuged at $100,000 \mathrm{~g}$ for $1 \mathrm{~h}$ at $4{ }^{\circ} \mathrm{C}$. The precipitate was collected and dissolved in homogenization buffer to obtain microsomes.

\section{ATPase activity measurement}

ATPase activities of MEF cells were measured using a colorimetric ATPase assay kit according to the manufacturer's protocol (Innova Biosciences, Cambridge, UK). Protein concentration was analyzed using the BCA assay (Thermo Scientific, Waltham, MA), and the ATPase activity was normalized by protein concentration.

\section{ER calcium store imaging}

Cells were incubated with $5 \mu \mathrm{mol} / \mathrm{L}$ Fura-2/AM (DOJINDO, Japan) in KREB buffer $(115 \mathrm{mmol} / \mathrm{L} \mathrm{NaCl}$, $4.7 \mathrm{mmol} / \mathrm{L} \mathrm{KCl}, 1.2 \mathrm{mmol} / \mathrm{L} \mathrm{MgSO}_{4} \cdot 7 \mathrm{H}_{2} \mathrm{O}, 1.2 \mathrm{mmol} / \mathrm{L}$ $\mathrm{KH}_{2} \mathrm{PO}_{4}, 25 \mathrm{mmol} / \mathrm{L} \mathrm{NaHCO}_{3}, 10 \mathrm{mmol} / \mathrm{L}$ HEPES, $2.5 \mathrm{mmol} / \mathrm{L} \mathrm{Ca}^{2+}$ ) with or without $25 \mathrm{mmol} / \mathrm{L}$ D-Glucose and $0.1 \%$ BSA at room temperature for $30 \mathrm{~min}$. For the starvation group, the cells were treated with Hanks buffer for $2 \mathrm{~h}$ followed by Fura-2/AM incubation. The culture medium was replaced with $0 \mathrm{mmol} / \mathrm{L} \mathrm{Ca}^{2+}$ KREB buffer followed by $2 \mu \mathrm{mol} / \mathrm{L} \mathrm{TG}$ treatment. Images were captured using an inverted microscope (IX81, Olympus, Japan) equipped with a $40 \times$ oil objective (numerical aperture $(\mathrm{NA})=1.3$, UPlanSApo, Olympus) and Zyla scientific complementary metal-oxide semiconductor (sCMOS) camera (ANDOR, Britain) at a rate of one frame per $800 \mathrm{~ms}$. The calcium concentration was represented by the ratio of $340 / 380$ of Fura-2/AM.

\section{$\mathrm{ER} \mathrm{Ca}^{2+}$ dynamic imaging}

Cells were transfected with GCEPIA1 $1_{\mathrm{ER}}$ or GCEPIA1SNAP $_{\text {ER }}$ using Lipofectamine 2000 (Invitrogen), and images were captured at a rate of one frame per 1 to $2 \mathrm{~s}$ using an FV1200 confocal microscope (OLYMPUS, Japan) equipped with a $60 \times$ oil objective lens (NA $=$ 1.35, UPlanSApo, Olympus). To display $\mathrm{Ca}^{2+}$ content at different time points or areas of the $z$-axis, a linear region of interest (ROI) over the time or $z$-axis area was plotted.

\section{Flow cytometry}

GCEPIA1-SNAP $_{\text {ER }}$ was transfected into MEF cells with Lipofectamine 2000 (Invitrogen). After 2 days, the cells were cultured in DMEM with or without $2 \mu \mathrm{mol} / \mathrm{L} \mathrm{TG}$ treatment for $1 \mathrm{~h}$, followed by incubation with $25 \mathrm{nmol} / \mathrm{L}$ SNAP-647 (NEB, USA) in an incubator at $37{ }^{\circ} \mathrm{C}$ with $5 \% \mathrm{CO}_{2}$ for $30 \mathrm{~min}$. And, finally, sorting by Flow Cytometry (FACSAriaIIIu, BD, USA). Data were acquired and analyzed on the FACSAriaIIIu with DIVA software (BD, USA).

\section{Statistics}

Data analysis was performed using OriginPro 8.1 software (OriginLab, USA). The unpaired $t$ test was used to determine the differences between nonstarvation and starvation groups. The values are presented as the mean \pm SD. The significant difference was set at $P<0.05$.

Acknowledgements This project was supported by the National Key R\&D Program of China (2016YFA0501500), the National Natural Science Foundation of China (31421002, 31401174 and 21778069), and the Project of the Chinese Academy of Sciences (XDB08030203). We thank Mr. Junying Jia from the Institute of Biophysics, Chinese Academy of Science for his help in FACS data collection.

\section{Compliance with Ethical Standards}

Conflict of interest Chen Luo, HuiyuWang, Qi Liu, Wenting He, Lin Yuan and Pingyong $\mathrm{Xu}$ declare that they have no conflict of interest.

Human and animal rights and informed consent This article does not contain any studies with human or animal subjects performed by the any of the authors. 
Open Access This article is distributed under the terms of the Creative Commons Attribution 4.0 International License (http:// creativecommons.org/licenses/by/4.0/), which permits unrestricted use, distribution, and reproduction in any medium, provided you give appropriate credit to the original author(s) and the source, provide a link to the Creative Commons license, and indicate if changes were made.

\section{References}

Akerboom J, Chen T-W, Wardill TJ, Tian L, Marvin JS, Mutlu S, Calderón NC, Esposti F, Borghuis BG, Sun XR (2012) Optimization of a GCaMP calcium indicator for neural activity imaging. J Neurosci 32:13819-13840

Carreras-Sureda A, Pihán P, Hetz C (2017) Calcium signaling at the endoplasmic reticulum: fine-tuning stress responses. Cell Calcium 70:24-31

Cho J-H, Swanson CJ, Chen J, Li A, Lippert LG, Boye SE, Rose K, Sivaramakrishnan S, Chuong C-M, Chow RH (2017) The GCaMP-R family of genetically encoded ratiometric calcium indicators. ACS Chem Biol 12:1066-1074

Clapham DE (2007) Calcium signaling. Cell 131:1047-1058

Cole NB (2013) Site-specific protein labeling with SNAP-tags. Curr Protoc Protein Sci. https://doi.org/10.1002/0471140864. ps3001s73

Ghislat G, Knecht E (2012) New $\mathrm{Ca}^{2+}$-dependent regulators of autophagosome maturation. Commun Integr Biol 5:308-311

Icha J, Weber M, Waters JC, Norden C (2017) Phototoxicity in live fluorescence microscopy, and how to avoid it. BioEssays 39:1700003

Koldenkova VP, Nagai T (2013) Genetically encoded $\mathrm{Ca}^{2+}$ indicators: properties and evaluation. Biochim Biophys Acta Mol Cell Res 1833:1787-1797

Liou J, Kim ML, Do Heo W, Jones JT, Myers JW, Ferrell JE Jr, Meyer T (2005) STIM is a $\mathrm{Ca}^{2+}$ sensor essential for $\mathrm{Ca}^{2+}$-storedepletion-triggered $\mathrm{Ca}^{2+}$ influx. Curr Biol 15:1235-1241

Mao T, O'Connor DH, Scheuss V, Nakai J, Svoboda K (2008) Characterization and subcellular targeting of GCaMP-type genetically-encoded calcium indicators. PLoS ONE 3:e1796
Park CY, Hoover PJ, Mullins FM, Bachhawat P, Covington ED, Raunser S, Walz T, Garcia KC, Dolmetsch RE, Lewis RS (2009) STIM1 clusters and activates CRAC channels via direct binding of a cytosolic domain to Orai1. Cell 136:876-890

Peinelt C, Vig M, Koomoa DL, Beck A, Nadler MJ, Koblan-Huberson M, Lis A, Fleig A, Penner R, Kinet J-P (2006) Amplification of CRAC current by STIM1 and CRACM1 (Orai1). Nat Cell Biol 8:771

Prakriya M, Feske S, Gwack Y, Srikanth S, Rao A, Hogan PG (2006) Orai1 is an essential pore subunit of the CRAC channel. Nature 443:230

Rizzuto R, Pinton P, Carrington W, Fay FS, Fogarty KE, Lifshitz LM, Tuft RA, Pozzan T (1998) Close contacts with the endoplasmic reticulum as determinants of mitochondrial $\mathrm{Ca}^{2+}$ responses. Science 280:1763-1766

Shigetomi E, Kracun S, Khakh BS (2010) Monitoring astrocyte calcium microdomains with improved membrane targeted GCaMP reporters. Neuron Glia Biol 6:183-191

Stutzmann GE (2005) Calcium dysregulation, IP3 signaling, and Alzheimer's disease. The Neuroscientist 11:110-115

Suzuki J, Kanemaru K, Ishii K, Ohkura M, Okubo Y, Iino M (2014) Imaging intraorganellar $\mathrm{Ca}^{2+}$ at subcellular resolution using CEPIA. Nat Commun 5:4153

Tian L, Hires SA, Mao T, Huber D, Chiappe ME, Chalasani SH, Petreanu L, Akerboom J, McKinney SA, Schreiter ER (2009) Imaging neural activity in worms, flies and mice with improved GCaMP calcium indicators. Nat Methods 6:875

Wäldchen S, Lehmann J, Klein T, Van De Linde S, Sauer M (2015) Light-induced cell damage in live-cell super-resolution microscopy. Sci Rep 5:15348

Zhang SL, Yu Y, Roos J, Kozak JA, Deerinck TJ, Ellisman $\mathrm{MH}$, Stauderman KA, Cahalan MD (2005) STIM1 is a $\mathrm{Ca}^{2+}$ sensor that activates CRAC channels and migrates from the $\mathrm{Ca}^{2+}$ store to the plasma membrane. Nature 437:902

Zhao Y, Araki S, Wu J, Teramoto T, Chang Y-F, Nakano M, Abdelfattah AS, Fujiwara M, Ishihara T, Nagai T (2011) An expanded palette of genetically encoded $\mathrm{Ca}^{2+}$ indicators. Science. https://doi.org/10.1126/science.1208592 